\title{
Surgical treatment of mono-segmental spinal tuberculosis using a combination of debridement and extreme lateral interbody fusion (XLIF): A retrospective study
}

\author{
Yi Zhan \\ Affiliated Hospital of Guilin Medical University https://orcid.org/0000-0002-3229-757X \\ Ke Chen \\ Fifth Affiliated Hospital of Guangzhou Medical University \\ Wuliang Huang \\ Fifth Affiliated Hospital of Guangzhou Medical University \\ Weijian Chen \\ Fifth Affiliated Hospital of Guangzhou Medical University \\ Yulin Lin ( $\square$ linyulin007@163.com) \\ Fifth Affiliated Hospital of Guangzhou Medical University
}

\section{Research article}

Keywords: Extreme lateral interbody fusion (XLIF), mono-segmental spinal tuberculosis, minimallyinvasive surgery

Posted Date: May 26th, 2020

DOI: https://doi.org/10.21203/rs.3.rs-29092/v1

License: (c) (i) This work is licensed under a Creative Commons Attribution 4.0 International License.

Read Full License 


\section{Abstract \\ Background}

This study investigated the clinical efficacy and safety of a combination of debridement and extreme lateral internal fusion (XLIF) for mono-segmental spinal tuberculosis (TB).

\section{Methods}

The medical records of 9 patients (aged 21-70 years; 6 males) with mono-segmental spinal TB treated at our hospital between January 2014 and November 2016 were retrospectively reviewed. The involved vertebral bodies included 2 cases each at the T6/7, L2/3 or L3/4 level and 1 case each at the T7/8, T8/9, or T9/10 level.

\section{Results}

All patients were successfully treated with a combination of debridement and XLIF. The mean length of the operation was $97.3 \pm 20.6 \mathrm{~min}$ (range, 65-126 min), and the mean blood loss during surgery was $151.1 \pm 25.7 \mathrm{ml}$ (range, 105-185 ml). Two patients experienced sensory disturbance over the left thigh and iliopsoas muscle weakness after surgery. After a mean follow-up of $12.3 \pm 3.7$ months (range, $6-17$ months), the mean Cobb angle decreased from $28.7 \pm 6.7$ degrees to $12.1 \pm 3.7$ degrees. The mean VAS pain score decreased from $7.2 \pm 1.1$ preoperatively to $2.3 \pm 0.9$ postoperatively. The mean bone graft fusion time was 5.6 months (range, 4.7-7.2 months). At the final follow-up, spinal cord injuries were evaluated as ASIA Grade D in 5 patients and ASIA Grade E in 4 patients.

\section{Conclusions}

A combination of debridement and XLIF is effective for the treatment of mono-segmental spinal TB, and is associated with minimal intraoperative trauma, few complications, and an improved quality of life for patients.

\section{Trial registration:}

This was a retrospective study. The study was approved by the institutional review board and the committee of our hospital. Informed consent to use their data was obtained from all patients before surgery.

\section{Background}


During the last two decades, advances in medical technologies have contributed to reducing the burden of tuberculosis (TB) worldwide. However, TB remains common in western China and certain other regions around the globe [1]. Skeletal TB occurs in approximately $10 \%$ of patients with extrapulmonary manifestations of the disease, and spinal TB accounts for an estimated $50 \%$ of cases of skeletal TB [2].

Anti-tuberculous chemotherapy alone has proven effective in patients with uncomplicated spinal TB, but surgical treatment is warranted when patients have obvious clinical manifestations such as bone destruction, abscesses, kyphosis, or spinal cord compression [2-7]. Surgical management for monosegmental spinal TB by traditional single-stage posterior or combined anterior and posterior has been described in the literature [5-7]. However, these techniques are associated with high rates of morbidities, including trauma, bleeding, and an extended recovery period.

Extreme lateral internal fusion (XLIF) was first described in 2006 by Ozgur et al. [8] as an approach for degenerative spinal diseases, and has been demonstrated to minimize blood loss, muscle damage, and surgical site pain [9]. However, the method has rarely been used for spinal TB or spondylodiscitis. In this study, we retrospectively reviewed the medical records of patients with mono-segmental spinal TB treated with a combination of debridement and XLIF in a one-stage operation.

\section{Materials And Methods}

\section{Study subjects}

The study protocol was approved by the Institutional Review Board of our Hospital. Patients with monosegmental spinal TB treated at our hospital between January 2014 and November 2016 were eligible for this study. Inclusion criteria were: 1 ) imaging findings (X-ray, CT, and MRI) showing typical signs of spinal TB; 2) TB involving $\leq 2$ spinal vertebrae; 3 ) lesions involving the anterior and middle column of the spine; 4) presence of abscesses, bone destruction, and spinal instability; and 5) TB confirmed on postoperative pathology. Exclusion criteria were: 1) contraindications to surgery; 2) TB involving > 2 vertebrae; or 3 ) severe osteoporosis.

\section{Preoperative procedure}

All patients received standard HREZ chemotherapy (300 mg/day isoniazid, $450 \mathrm{mg} /$ day rifampicin, 750 $\mathrm{mg} /$ day ethambutol, $750 \mathrm{mg} /$ day pyrazinamide) and nutritional support for 2-3 weeks before the procedure. Surgery was performed when patients had recovered from anemia, hypoproteinemia, and neurological symptoms and their erythrocyte sedimentation rate (ESR) and body temperature were normal.

\section{Operative procedure}

After administration of anesthesia with standard single lumen endotracheal intubation, patients were placed in a 90 degree lateral position and the lesion segment of the vertebral body and the skin incision were marked using fluoroscopy. An oblique incision (about 4-6 cm) was made in the median axillary line 
above the rib to avoid injuring the neurovascular bundle. Under real-time $\mathrm{C}$-arm fluoroscopy, the lung was pushed away using finger dissection, and a dilator was placed directly against the vertebral column at the surgical level (Figure 1). At the lumbar level, the oblique externus abdominis and obliquus internus abdominis were bluntly separated to provide access to the retroperitoneal space, the peritoneum was pushed forward in front of the psoas major muscle with finger dissection, and sequential dilation was performed with progressively larger dilators (Figure 2). A guide wire was inserted into the middle or anterior part of the diseased intervertebral space to avoid injuring the spinal cord or nerve root. C-arm fluoroscopy was used to confirm the position of the dilator. One-step lesion debridement was used to drain the prevertebral abscess and remove diseased bone. The length of the bone grafting groove was measured. The same length of iliac bone was implanted into the bone groove and fixed with an anterior plate and screw. Streptomycin $(1 \mathrm{~g})$ and isoniazid $(0.5 \mathrm{~g})$ were administered around the bone graft. A drainage tube was placed and the incision was sutured. The debrided material underwent histopathologic examination.

\section{Postoperative care}

Antibiotics were administered for $72 \mathrm{~h}$ after surgery. The drainage tube was removed when drainage volume was $<50 \mathrm{ml} /$ day. Standard anti-TB drugs were administered for 12-18 months, and liver and kidney function and ESR were monitored at regular intervals. After one week of bed rest, patients were permitted to perform ambulatory activities wearing a brace, which was worn for at least 6 months after surgery.

\section{Curative efficacy evaluation}

Plain radiographs and CT of the spine were obtained one week after surgery and every three months thereafter. Patients were evaluated based on American Spinal Injury Association (ASIA) grading, the visual analogue scale (VAS) pain score and Cobb angle, which were recorded at one month after surgery and the final follow up. Suk's criteria were used to assess bone graft fusion [10].

\section{Results}

This study included 9 patients ( 6 men and 3 women) with mono-segmental spinal TB. Preoperatively, patient's mean age was 41.1 years (range 21-70 years), and mean body mass index (BMI) was $22.1 \mathrm{~kg} / \mathrm{m}^{2}$. All patients experienced the following symptoms: fever, night sweats, back pain, and weight loss. Spinal cord injuries were evaluated as ASIA Grade B in 2 patients, ASIA Grade $C$ in 3 patients, ASIA Grade $D$ in 3 patients, and ASIA Grade E in 1 patient. The involved vertebral bodies included 2 cases each at the T6/7, L2/3 or L3/4 level and 1 case each at the T7/8, T8/9, or T9/10 level (Table 1 ).

All surgeries were successful. Mean operative time was $97.3 \pm 20.6 \mathrm{~min}$ (range, 65-126 min), and mean blood loss during surgery was $151.1 \pm 25.7 \mathrm{ml}$ (range, 105-185 ml). There were no complications, such as incision infection, recurrence of TB, pleural effusions, rib pain, or delayed pneumothorax, and there 
were no fatalities. Two patients experienced sensory disturbance over the left thigh and iliopsoas muscle weakness after surgery. They were provided symptomatic treatment and recovered within 3 months.

All patients were followed up for a mean of $12.3 \pm 3.7$ months (range, $6-17$ months). Mean Cobb angle decreased from $28.7 \pm 7.1$ degrees preoperatively to $12.1 \pm 3.7$ degrees postoperatively. Mean VAS pain score decreased from $7.2 \pm 1.1$ preoperatively to $2.3 \pm 0.9$ postoperatively (Table 2 ).

Mean bone graft fusion time was 5.6 months (range, 4.7-7.2 months) (Figs. 3 and 4). At final follow-up, spinal cord injuries were evaluated as ASIA Grade D in 5 patients and ASIA Grade E in 4 patients (Table 3).

\section{Discussion}

Our data demonstrates that spinal TB can be effectively treated using debridement and XLIF during a single operation. The technique made it possible to retain the spine's stability, while minimizing the length of the operation and blood loss, with few complications. As detailed above, patients had improvements in both their VAS scores and postoperative neurologic recovery.

Compared with laparoscopic or thoracoscopy-assisted approaches for the treatment of mono-segmental spinal TB, the XLIF approach has several advantages. First, XLIF eliminates the need for a general surgical assistant and the steep learning curve associated with laparoscopic or thoracoscopic approaches $[8,11]$. Second, the mean length of the operation and blood loss in our patients were significantly minimized compare with previous series of video-assisted thoracoscopic surgery for spinal TB described by Lv et al. and Kapoor et al. [11, 12]. Third, a long period of lung deflation can be avoided, which makes XLIF ideal for use in elderly patients or those with respiratory compromise $[13,14]$. Finally, the technique allows direct visualization, which is particularly helpful for removing the infected tissue [15].

Perhaps most importantly, the stability of the spine was retained. Although there was a decrease in the Cobb angle, the average correction rate of the kyphotic angle was $58.0 \%$. Biomechanical studies $[16,17]$ indicated that transpedicular fixation conferred better stabilization than lateral fixation, but both methods can provide adequate stability. The major disadvantage of transpedicular fixation combined with XLIF is that it prolongs the operation. Timothy et al. [15] reported that posterior instrumentation was not necessary, especially in the setting of infection, because of the high bone fusion rate associated with using XLIF. In addition, mono-segmental fixation can retain the spinal motion units and slow the degeneration of adjacent vertebral bodies. The average bone fusion time was $5.6 \pm 0.8$ months, which was similar to other studies $[5-7,13,14]$. Moreover, the quality of life improved significantly in accordance with the better VAS score and neurological improvement.

Notably, surgeons should be aware of the possible neurological complications associated with XLIF. In the present study, two patients experienced sensory disturbance over the left thigh and iliopsoas muscle weakness after surgery. The patients recovered following three months of physical therapy and oral 
methylcobalamin. A nationwide survey conducted between March 2013 and April 2015 in Japan reported that the most common complications associated with XLIF were sensory nerve injury (5.1\%) and psoas weakness (4.3\%) [18]. Tohmeh et al. [19] proposed the use of dynamically-evoked electromyography (EMG) to detect and prevent neural injury during XLIF. We suggest that surgeons should have accurate knowledge of the spinal nerve rather than relying on EMG to avoid potential neurological complications $[20,21]$.

Based on our clinical experience, we recommend using a combination of debridement and XLIF for the treatment of mono-segmental spinal TB without a large abscess or severe bone defects. The operating area and the guide needle puncture should be in front of the psoas muscle and vertebra, respectively. Standardized anti-TB treatment and regular follow up are very important. Patients should be made aware of potential complications, including delayed pneumothorax or hemothorax, vascular injury, and nonpulmonary complications, so that they can be carefully monitored postoperatively $[13,14]$.

This study has several potential limitations. First, the results are from a relatively small sample size and there was no control group. Second, the follow-up was relatively short. Third, the indications for the procedure were based on our experience. Additional multicenter prospective studies with a longer followup are necessary to better characterize the efficacy and safety of XLIF for the treatment of spinal TB.

\section{Conclusion}

A combination of debridement and XLIF is effective for the treatment of mono-segmental spinal TB as it is associated with minimal intraoperative trauma, few complications, and improvements in the patient quality of life.

\section{Abbreviations}

ASIA, American Spinal Injury Association; TB, tuberculosis; VAS, visual analog scale; XLIF, extreme lateral interbody fusion

\section{Declarations}

\section{Ethics approval and consent to participate}

The authors are accountable for all aspects of the work in ensuring that questions related to the accuracy or integrity of any part of the work are appropriately investigated and resolved. The study was approved by the institutional review board and the committee of our hospital.

\section{Consent for publication}

Informed consent to use their data was obtained from all patients before surgery.

\section{Availability of data and materials}


The datasets used and/or analysed during the current study are available from the corresponding author on reasonable request.

\section{Competing interests}

The authors have no competing interests to disclose.

\section{Funding}

None

\section{Authors' contributions}

Yi Zhan and Yulin Lin participated in the conception and design. Yulin Lin provided adiministrative support. Ke Chen and Wuliang Huang took charge of provision of study materials or patients. Ke Chen and Weijian Chen took charge of collection and assembly of data. Yi Zhan and Yulin Lin took charge of data analysis and interpretation. All authors wrote and approved the final manuscript.

\section{Acknowledgements}

Not applicable.

\section{References}

1. Wang L, Zhang H, Ruan Y, et al. Tuberculosis prevalence in China, 1990-2010; a longitudinal analysis of national survey data. Lancet. 2014;383:2057-64.

2. Fuentes Ferrer M, Gutierrez Torres $L$, Ayala Ramirez $O$, et al. Tuberculosis of the spine. A systematic review of case series. Int Orthop. 2012;36:221-31.

3. Wang LJ, Zhang HQ, Tang MX, et al. Comparison of Three Surgical Approaches for Thoracic Spinal Tuberculosis in Adult: Minimum 5-Year Follow Up. Spine. 2017;42:808-17. doi:10.1097/ BRS.0000000000001955.

4. Liu J, Wan L, Long X, et al. Efficacy and Safety of Posterior Versus Combined Posterior and Anterior Approach for the Treatment of Spinal Tuberculosis: A Meta-Analysis. World Neurosurg. 2015;83:1157-65. doi:10.1016/j.wner.2015.01.041.

5. Xu Z, Wang X, Wu P, et al. Surgical treatment for mono-segmental lumbar tuberculosis by singlestage posterior debridement, compact bone grafting and posterior single-segment fixation. Injury. 2015;46:1311-6. doi:10.1016/j.injury.2015.03.023.

6. Wang Z, Wu Q, Geng G. Anterior debridement and bone grafting with posterior single-segment internal fixation for the treatment of mono-segmental spinal tuberculosis. Injury. 2013;44:253-7. doi:10.1016/j.injury.2012.11.003.

7. Wang Z, Yuan H, Geng G, et al. Posterior mono-segmental fixation, combined with anterior debridement and strut graft, for treatment of the mono-segmental lumbar spinal tuberculosis. Int 
Orthop. 2012;36:325-9. doi:10.1007/s00264-011-1475-4.

8. Ozgur BM, Aryan HE, Pimenta L, et al. Extreme Lateral Interbody Fusion (XLIF): a novel surgical technique for anterior lumbar interbody fusion. Spine J. 2006;6:435-643.

9. Lang G, Perrech M, Navarro-Ramirez R, et al. Potential and limitations of Neural Decompression in Extreme Lateral Interbody Fusion-A systematic Review. World Neurosurg. 2017;101:99-113. doi:10.1016/j.wneu.2017.01.080.

10. Suk SI, Lee CK, Kim WJ, et al. Adding posterior lumbar interbody fusion to pedicle screw fixation and posterolateral fusion after decompression in spondylolytic spondylolisthesis. Spine 1997;22:210-9; discussion 9-20.

11. Lv GH, Wang B, Li J, et al. Thoracoscopy-assisted mini-open surgery for anterior column reconstruction in thoracic spinal tuberculosis. Orthop Surg. 2009;1:293-9. doi:10.1111/j.17577861.2009.00043.x.

12. Kapoor SK, Agarwal PN, Jain BK Jr, et al. Video-assisted thoracoscopic decompression of tubercular spondylolytic: clinical evaluation. Spine 2005;30:E605-10.

13. $10.1007 / \mathrm{s} 11420-012-9312-x$

Meredith DS, Kepler CK, Huang RC, et al. Extreme Lateral Interbody Fusion (XLIF) in the Thoracic and Thoracolumbar Spine: Technical Report and Early Outcomes. HSS J 2013;9:25-31. doi: 10.1007/s11420-012-9312-x.

14. Karikari IO, Nimjee SM, Hardin CA, et al. Extreme lateral interbody fusion approach for isolated thoracic and thoracolumbar spine diseases: initial clinical experience and early outcomes. J Spinal Disord Tech. 2011;24:368-75. doi:10.1097/BSD.0b013e3181ffefd2.

15. Timothy J, Pal D, Akhunbay-Fudge C, et al. Extreme lateral interbody fusion (XLIF) as a treatment for acute spondylodiscitis: Leeds spinal unit experience. J Clin Neurosci. 2019;59:213-7.

16. Laws CJ, Coughlin DG, Lotz JC, et al. Direct lateral approach to lumbar fusion is a biomechanically equivalent alternative to the anterior approach: an in vitro study. Spine 2012;37:819-25. doi: 10.1097/ BRS.0b013e31823551aa.

17. Kim SM, Lim TJ, Paterno J, et al. Biomechanical comparison: stability of lateral-approach anterior lumbar interbody fusion and lateral fixation compared with anterior-approach anterior lumbar interbody fusion and posterior fixation in the lower lumbar spine. J Neurosurg Spine. 2005;2:62-8.

18. Fujibayashi S, Kawakami N, Asazuma T, et al. Complications Associated With Lateral Interbody Fusion: Nationwide Survey of 2998 Cases During the First 2 Years of Its Use in Japan. Spine 2017;42:1478-84. doi: 10.1097/ BRS.0000000000002139.

19. Tohmeh AG, Rodgers WB, Peterson MD. Dynamically evoked, discrete-threshold electromyography in the extreme lateral interbody fusion approach. J Neurosurg Spine. 2011;14:31-7. doi:10.3171/2010.9.SPINE09871.

20. Sun JC, Wang JR, Luo T, et al. Surgical Incision and Approach in Thoracolumbar Extreme Lateral Interbody Fusion Surgery: An Anatomic Study of the Diaphragmatic Attachments. Spine. 2016;41:E186-90. doi:10.1097/BRS.0000000000001183. 
21. Hu WK, He SS, Zhang SC, et al. An MRI study of psoas major and abdominal large vessels with respect to the X/DLIF approach. Eur Spine J. 2013;20:557-62. doi:10.1007/s00586-010-1609-1.

\section{Tables}

Table 1. Patients' demographic and clinical characteristics

\begin{tabular}{|c|c|c|c|c|c|c|c|c|}
\hline Case & Age & Gender & Lesion segment & OT, $\min$ & BMI & Blood loss, ml & FT (months) & F/U (months) \\
\hline 1 & 25 & M & $\mathrm{T} 7 / 8$ & 126 & 22.8 & 135 & 5.5 & 11 \\
\hline 2 & 36 & M & $\mathrm{T} 6 / 7$ & 106 & 24 & 180 & 6.1 & 17 \\
\hline 3 & 45 & $\mathrm{~F}$ & $\mathrm{~T} 6 / 7$ & 96 & 19.6 & 150 & 7.2 & 14 \\
\hline 4 & 27 & M & $\mathrm{T} 8 / 9$ & 115 & 22 & 175 & 5.0 & 6 \\
\hline 5 & 33 & $\mathrm{~F}$ & $\mathrm{~T} 9 / 10$ & 78 & 25 & 185 & 4.8 & 8 \\
\hline 6 & 62 & M & $\mathrm{L} 2 / 3$ & 106 & 20.8 & 125 & 4.7 & 17 \\
\hline 7 & 21 & M & $\mathrm{L} 2 / 3$ & 65 & 25.3 & 140 & 5.3 & 12 \\
\hline 8 & 70 & M & $\mathrm{L} 3 / 4$ & 69 & 20.1 & 165 & 5.2 & 16 \\
\hline 9 & 51 & $\mathrm{~F}$ & $\mathrm{~L} 3 / 4$ & 115 & 19.6 & 105 & 6.2 & 10 \\
\hline Mean & $41.1 \pm 16.1$ & & & $97.3 \pm 20.6$ & $22.1 \pm 2.1$ & $151.1 \pm 25.7$ & $5.6 \pm 0.8$ & $12.3 \pm 3.7$ \\
\hline
\end{tabular}

Abbreviations: OT, operation time; BMI, body mass index; FT, fusion time; FU, follow-up

Table 2. Postoperative outcomes

\begin{tabular}{cccccccc}
\hline \multicolumn{3}{c}{ Cobb angle $\left(^{\circ}\right)$} & & \multicolumn{2}{c}{ VAS score } \\
\hline Pre & 1 month post & FFU & Correction rate (\%) & Pre & 1 month post & FFU & Rate of improvement (\%) \\
\hline $28.7 \pm 6.7$ & $9.9 \pm 3.8$ & $12.1 \pm 3.7$ & $58.0 \%$ & $7.2 \pm 1.1$ & $4.1 \pm 0.9$ & $2.3 \pm 0.9$ & $67.6 \%$ \\
\hline
\end{tabular}

Abbreviations: VAS, visual analogue scale; FFU, final follow-up

Table 3. Neurological outcomes 


\begin{tabular}{|c|c|c|c|c|c|c|}
\hline \multirow[t]{2}{*}{ Preoperative } & \multirow[t]{2}{*}{$\mathbf{n}$} & \multicolumn{5}{|c|}{ Postoperative ASIA grade } \\
\hline & & A & B & $\mathrm{C}$ & $\mathrm{D}$ & $\mathrm{E}$ \\
\hline \multicolumn{7}{|l|}{$\mathbf{A}$} \\
\hline B & 2 & & & & 2 & \\
\hline C & 3 & & & & 2 & 1 \\
\hline D & 3 & & & & 1 & 2 \\
\hline $\mathbf{E}$ & 1 & & & & & 1 \\
\hline
\end{tabular}

Abbreviations: ASIA, American Spinal Injury Association

\section{Figures}

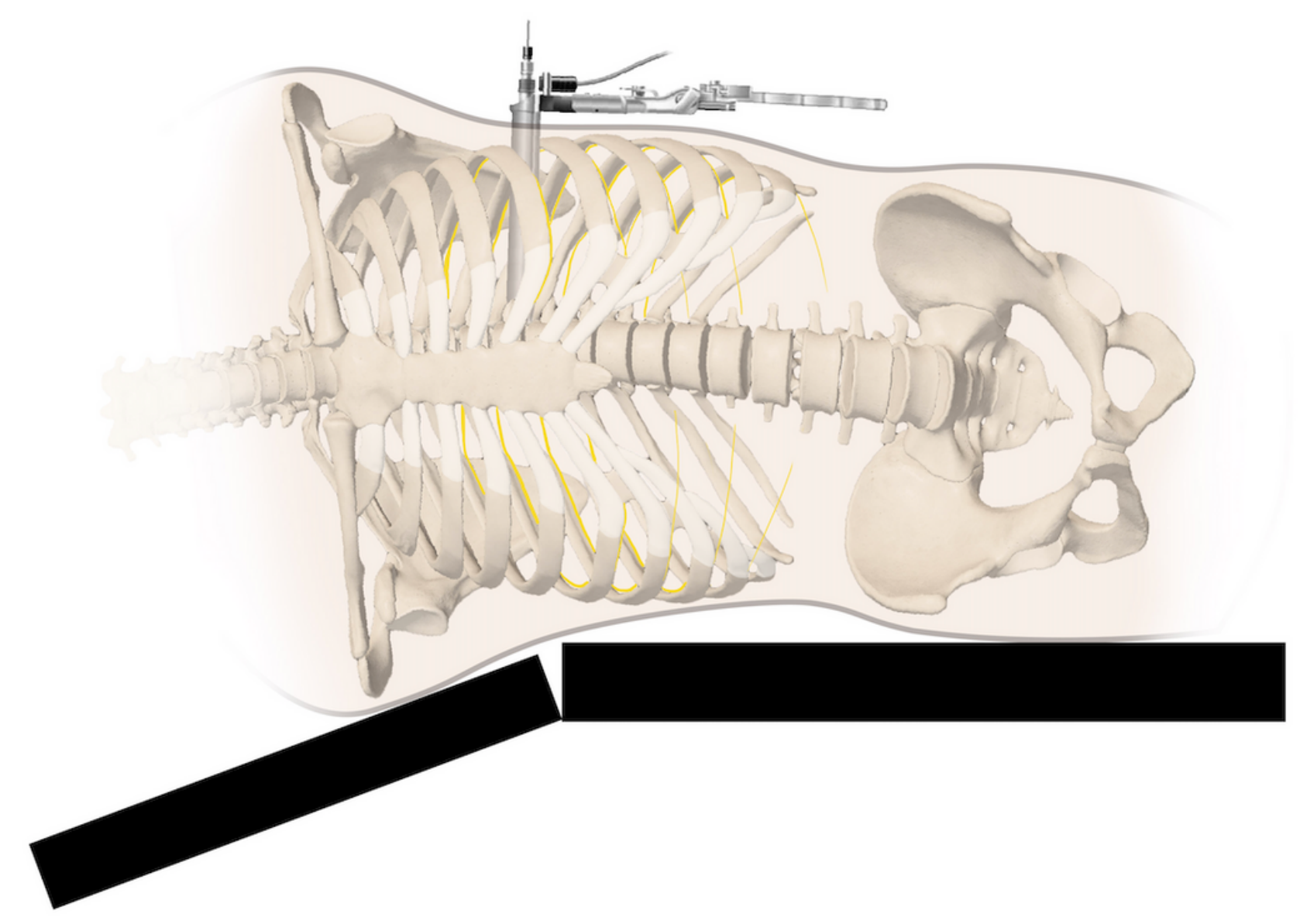

Figure 1

Illustration of the patient's posture and the XLIF approach in the thoracic spine 


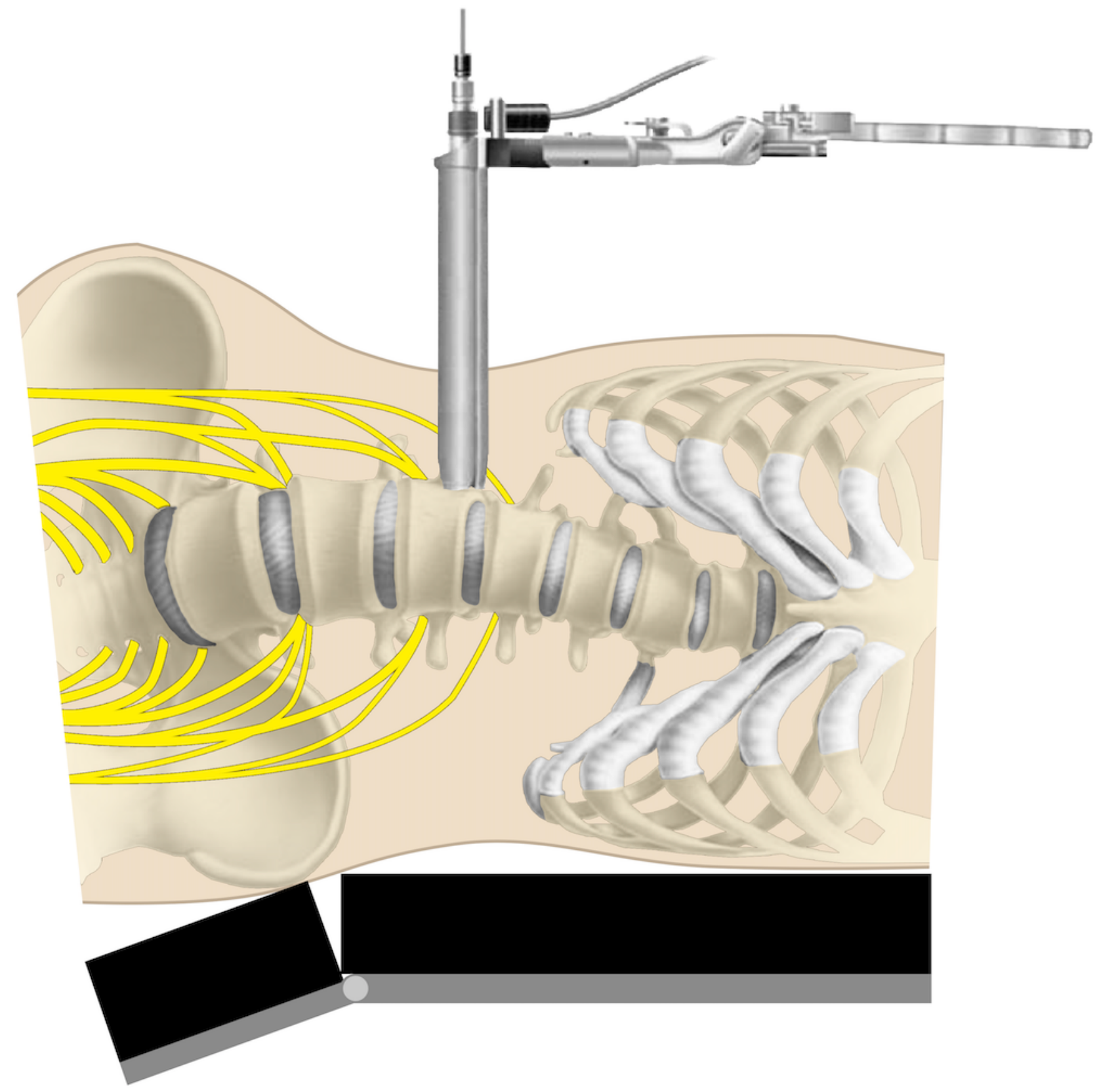

Figure 2

Illustration of the patient's posture and the XLIF approach in the lumbar spine 

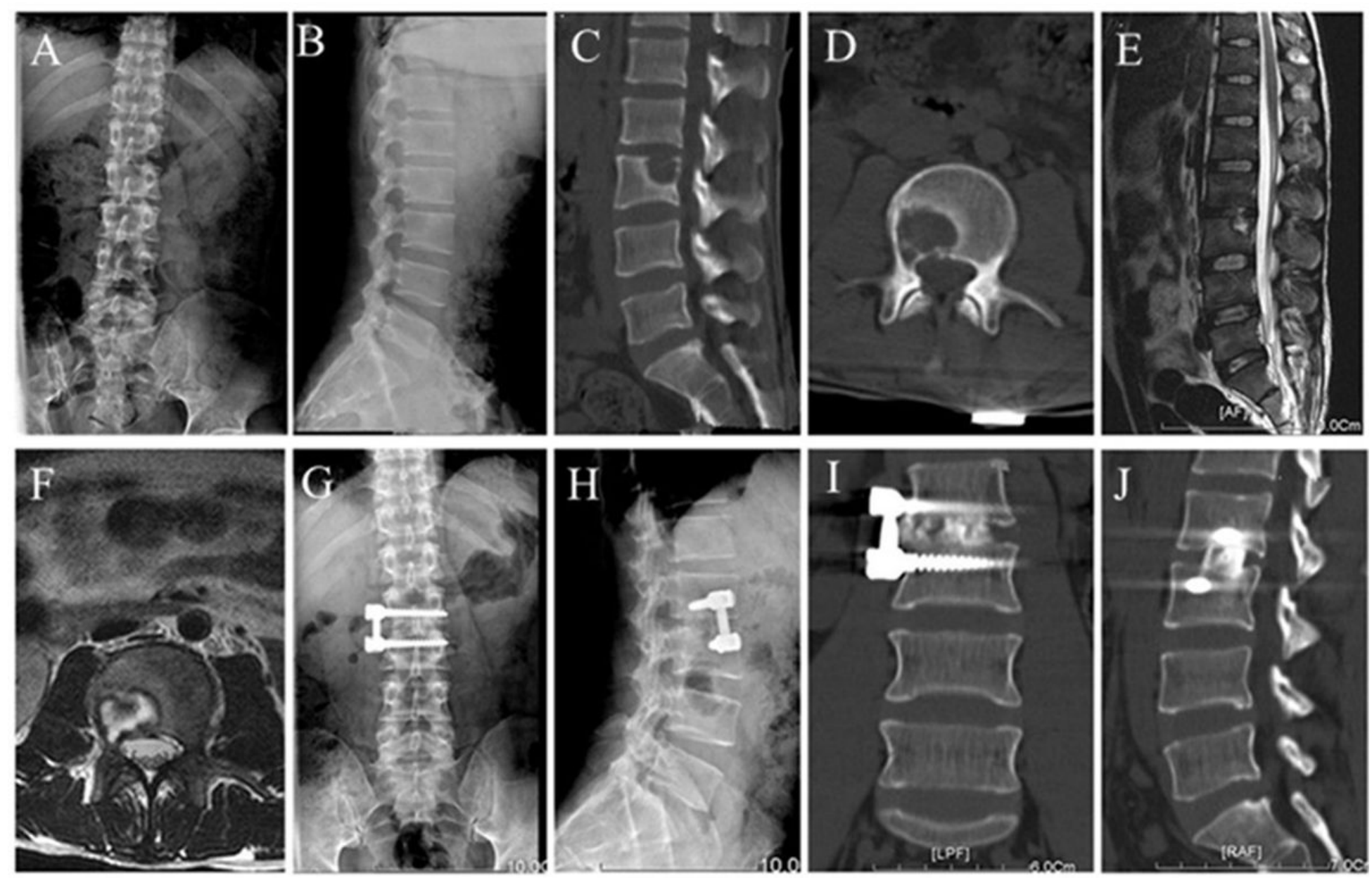

Figure 3

A 21-year-old male with L2/3 TB lesions and bone destruction. (A,B) Preoperative anteroposterior-lateral radiograph; (C,D) Preoperative sagittal and transverse $C T$; $(E, F)$ Preoperative MRI. $(G, H)$ Postoperative anteroposterior-lateral radiograph. $(\mathrm{l}, \mathrm{J}) 6$ month postoperative CT showing internal fixation was in a good position and bone fusion. 


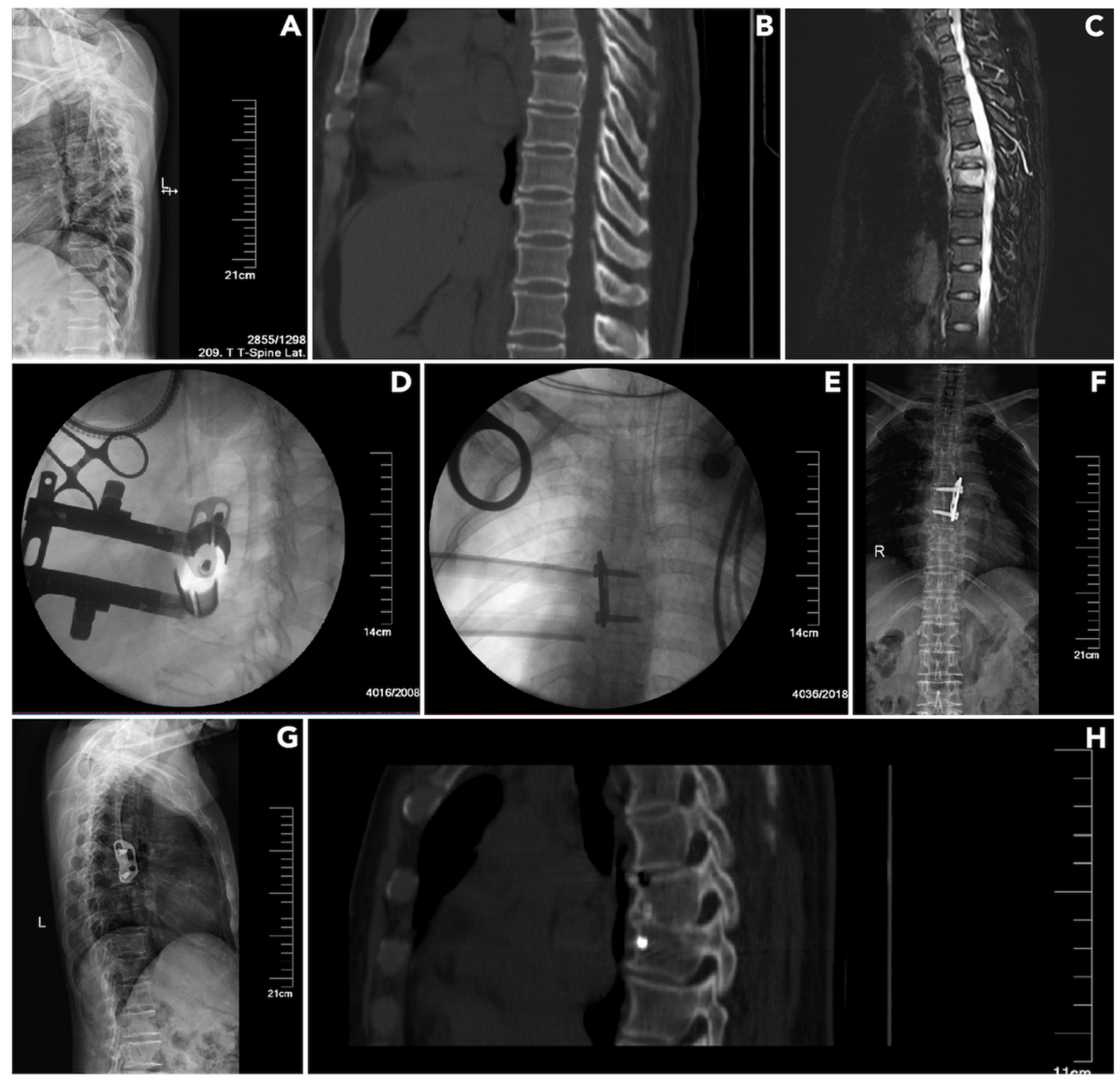

Figure 4

A-C, 45-year-old female with T6/7 TB lesions and bone destruction. (A-C) Preoperative X-ray, CT, and MRI confirmed spinal TB at the T6/7 level. (D-E) The patient was treated with a combination of debridement and XLIF. (F-H) At the 10 month follow-up, X-ray showed no lateral curvature or kyphosis, and CT showed good bone fusion. 